\title{
Split Peroneus Brevis Tendon: An Unusual Cause of Ankle Pain and Instability
}

\author{
Bindiya Chauban, MD, Pina Panchal, MD, Edward Szabo, DPM, \\ and Thad Wilkins, MD
}

Tears of the peroneus brevis tendon may cause ankle pain, swelling, and instability. Supportive therapy with ankle bracing and analgesics is the mainstay of therapy, but surgical repair is often required in patients with ongoing symptoms. Surgical options include debridement, tubularization, or, in severe cases, resection of the damaged tendon and tenodesis. We describe a 64-year-old woman with a split peroneus brevis tendon presenting with lateral ankle pain, swelling, and instability, and we review the literature regarding presentation, diagnostic testing, pathophysiology, predisposing factors, and treatment recommendations. Primary care physicians should consider peroneal tendon injuries in patients with chronic lateral ankle pain and instability. (J Am Board Fam Med 2014;27:297-302.)

Keywords: Ankle, Case Reports, Joint Instability, Pain, Tendons, Treatment

A 64-year-old white woman presented to our family medicine clinic with complaints of right lateral ankle pain with dorsiflexion and inability to bear weight for the past 4 weeks. Her inability to bear weight was related to the sensation of instability. She denied any recent trauma or injury to her ankle or associated symptoms, including swelling, bruising, numbness, weakness, or tingling. She reported a "severe" ankle sprain 20 years ago while running, which required casting for 6 weeks. After the cast was removed, she continued to have pain and required multiple corticosteroid injections. She continued to have pain for about 5 years after her injury; she reported that the pain had resolved until 4 weeks before her office visit, when the pain returned. She denied using tobacco, alcohol, or illicit drugs. Her family history was negative for cancers or orthopedic problems. Her medications included aspirin, calcium, cholestyramine, diclofenac topical, hydralazine, losar-

This article was externally peer reviewed.

Submitted 14 March 2013; revised 31 July 2013; accepted 12 August 2013.

From the Department of Family Medicine (BC, PP, TW) and the Department of Orthopedics (ES), Medical College of Georgia, Georgia Regents University, Augusta.

Funding: none.

Conflict of interest: none declared.

Corresponding author: Thad Wilkins, M.D., Department of Family Medicine, Medical College of Georgia, Georgia Regents University, 1120 15th Street, HB-4032, Augusta, GA 30912 (E-mail: jwilkins@gru.edu). tan/hydrochlorothiazide, spironolactone, metformin, metoprolol succinate, minocycline, multivitamin, omega-3 fatty acids, oxycodone/acetaminophen, sulindac, and vitamin $\mathrm{B}_{12}$.

During her examination, her height was 61 in, weight was $173 \mathrm{lb}$, and body mass index was 32.8 $\mathrm{kg} / \mathrm{m}^{2}$. She appeared well nourished and in no distress. There was extensive lateral ankle swelling but no bruising. She was unable to completely bear her weight on her right foot secondary to pain, and she had tenderness on the lateral malleolus. The drawer test and talar tilt were not performed because of the pain. A radiograph was obtained and showed widening of the lateral talotibial joint, suggesting some lateral ligamentous instability (Figure 1). She was prescribed crutches and an air splint. Sulindac was discontinued and she was started on naproxen.

She returned 2 weeks later with persistent lateral ankle pain and the sensation that the ankle was going to "give out." Magnetic resonance imaging (MRI) of the right ankle showed that the peroneal brevis tendon was flattened to the borders of both the medial and lateral aspects of the peroneal longus, consistent with a split tendon (Figure 2). Ankle joint effusion also was present. We chose MRI instead of computed tomography (CT) after radiograph because we did not suspect an occult fracture (she had no recent history of trauma or injury). She was referred for physical therapy but complained of 
Figure 1. Oblique radiograph showing widening of the lateral talotibial joint (arrows), suggesting some lateral ligamentous instability.



persistent ankle pain and no improvement of her symptoms after physical therapy. She was advised to wear heel pads and ankle support with a lace-up ankle brace. She was referred to a podiatrist, who recommended surgical repair or prolonged immobilization with conservative management. At this time, our patient has declined surgery.

\section{Discussion}

Ankle sprains are the most common musculoskeletal injury, occurring in 1 in 10,000 people daily, and symptoms are chronic in $40 \%$ of patients. ${ }^{1,2}$ Ankle sprains account for $16 \%$ to $21 \%$ of all exercise-related injuries. ${ }^{1}$ Young adults and teenagers have a higher incidence of ankle sprains because they are often more active. ${ }^{3}$ One in 5 of all sports injuries are ankle sprains, with basketball being the number one sport for causing this injury. ${ }^{1,3,4}$ Ankle instability is a complication of ankle sprains and is caused by ligamentous injury. It is associated with recurrent injuries and repetitive sprains. ${ }^{5,6}$ Ankle injuries can affect medial and lateral ligaments, but lateral ligament injuries are more common: $85 \%$ of ankle injuries involve the lateral ligaments. ${ }^{1}$ Problems associated with lateral ligament injuries include peroneal tendon injuries, hindfoot varus alignment abnormalities, retinacular pathology, and anterolateral impingement lesions. ${ }^{7,8}$ Ligamentous injuries may lead to chronic ankle instability, which increases the risk of recurrent sprains and additional injury. ${ }^{6}$ The duration of ankle insta- bility and pain are related to the increased likelihood of having a split or tear of the ligament. ${ }^{9}$

A cause of lateral ankle pain and instability is a split or tear of the peroneus brevis tendon (PBT) or peroneus longus tendon (PLT); however, PBT tears are more common. Split lesions of the PBT were thought to be relatively rare; however, a study of 112 cadaveric ankles found a prevalence of $37.5 \%$ based on thinning or longitudinal splitting of PBT. ${ }^{10,11}$ The peroneus can split because of the stress of stretching that occurs from the inversion of the lateral ankle injury. The PLT overlies and presses on the PBT, possibly resulting in a split. Other causes of PBT or PLT splits or tears include chronic instability or external compression from anatomic variants causing repetitive friction of the tendon over the posterolateral fibula, resulting in the tear.

\section{Literature Search}

We searched PubMed and Ovid using the search term split peroneus brevis tendon. We found no randomized controlled trials, but found 1 systematic review, 3 clinical reviews, 2 retrospective studies, 2 longitudinal studies, and 1 case series. In addition, we searched ankle injuries, peroneal tendonopathies, peroneal brevis tears, peroneus longus tears, Ottawa ankle rules, talar tilt test, and anterior drawer test.

Figure 2. Proton density-weighted axial magnetic resonance image demonstrating one of the appearances of a split peroneus brevis tendon splayed over the normal-appearing peroneus longus tendon. Here, the peroneal brevis tendon was flattened and borders both the medial and lateral aspects of the peroneal longus, consistent with a split tendon.

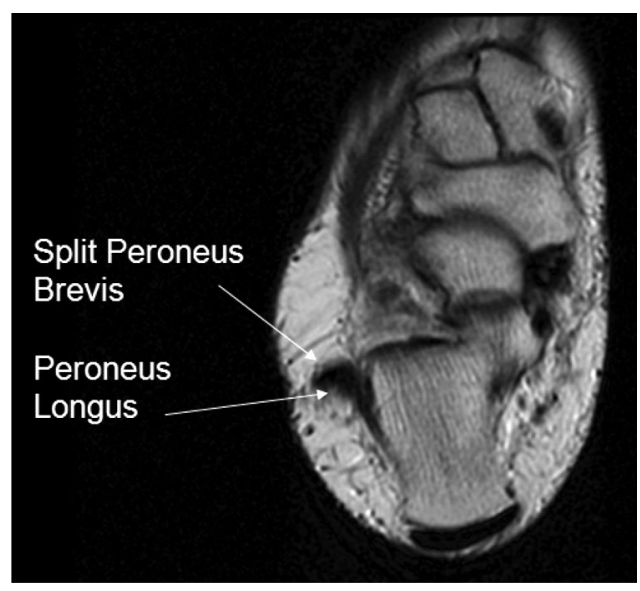


Table 1. A Grading System for Split Peroneus Brevis Tendon

\begin{tabular}{|c|c|c|c|}
\hline Grade of Split & Description & Magnetic Resonance Imaging Findings & Treatment \\
\hline I & Splayed-out tendon & $\begin{array}{l}\text { The peroneus brevis tendon is flattened between } \\
\text { the peroneus longus tendon and lateral } \\
\text { malleolus. }\end{array}$ & Conservative treatment \\
\hline II & Partial-thickness split & $\begin{array}{l}\text { The tendon is flattened and also shows central } \\
\text { thinning and some degeneration of tendon } \\
\text { structure. }\end{array}$ & Conservative treatment \\
\hline III & Full-thickness split & $\begin{array}{l}\text { The tendon had a central "fenestration" and } \\
\text { appeared to be separated into } 2 \text { fragments on } \\
\text { a variable of section. }\end{array}$ & Surgical evaluation and repair \\
\hline IV & Split extending $>2 \mathrm{~cm}$ & $\begin{array}{l}\text { The tendon had a central "fenestration" and } \\
\text { appeared to be separated into } 2 \text { fragments on } \\
\text { a variable of section. }\end{array}$ & Surgical evaluation and repair \\
\hline
\end{tabular}

Figure 3. Algorithm for the management and treatment of suspected split peroneal brevis tendon.

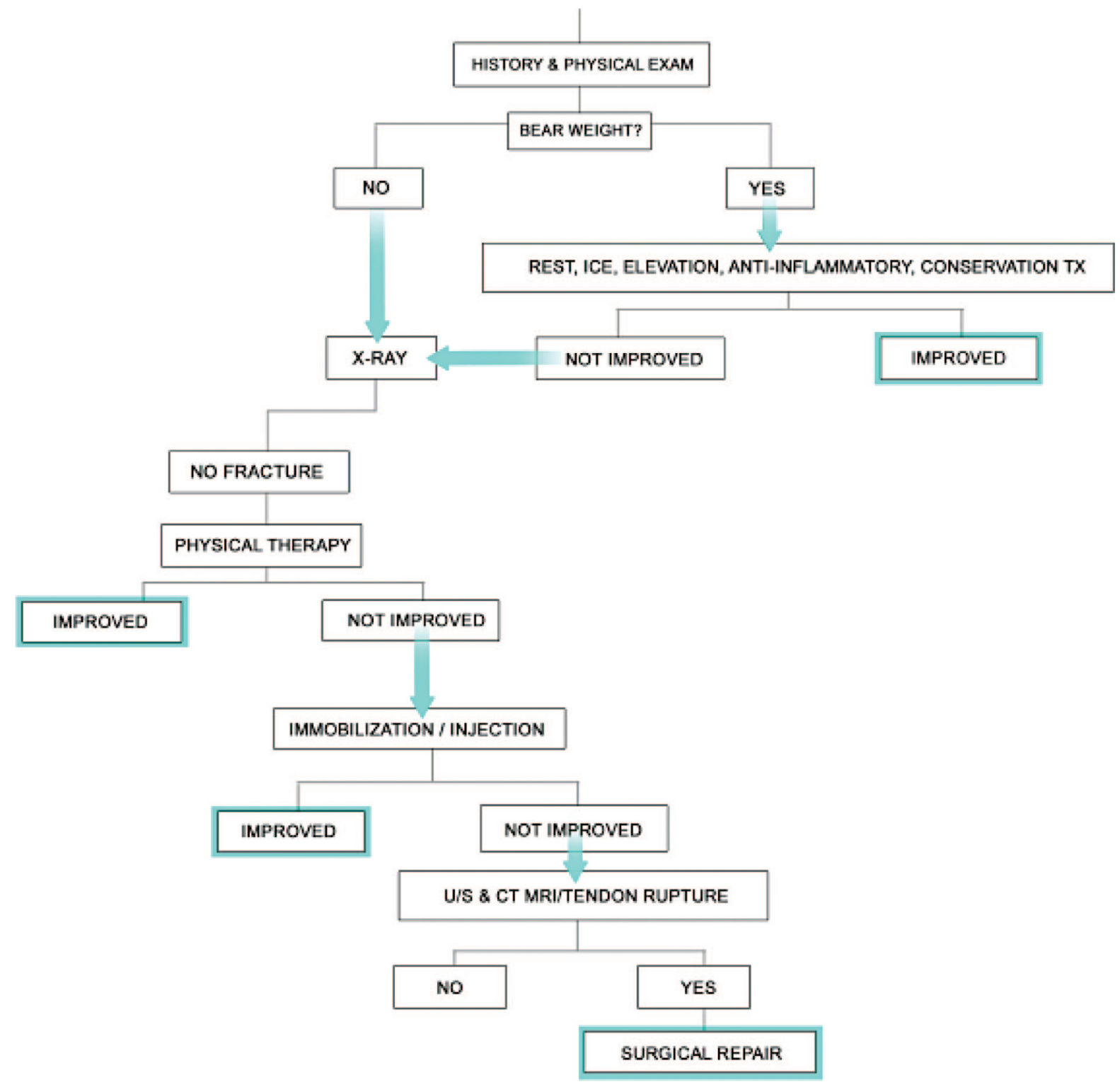




\section{Mechanism of Injury}

PBT tears often are associated with a forced dorsiflexion injury and are most commonly longitudinal rather than transverse. ${ }^{10} \mathrm{With}$ repetitive trauma or contraction of the peroneal muscles, the PBT may split. Splitting of the PBT can be caused by misalignment of the fibula following an ankle injury. ${ }^{12} \mathrm{~A}$ tear in the lateral PBT may occur after the superior peroneal retinaculum is torn when the ankle is everted during trauma. As the split progresses, the tendon is compressed between the lateral ridge of the malleolus and the peroneus longus tendon. See Table 1 for a grading scale for split PBT.

\section{Diagnostic Testing}

\section{Physical Examination}

A complete history and physical examination is the key to diagnosing the etiology of ankle pain and instability. A history includes asking about the onset of pain, mechanism of injury, duration and location of pain, clicking, sensation of the ankle "giving out," persistent or shooting pain, numbness, and tingling. The history is helpful to differentiate neuropathic, musculoskeletal, and vascular pain. Another important question to include is whether the patient could bear weight immediately following the injury and if they can do so during the evaluation (Figure 3). Physical examination consists of inspection, palpation for tenderness, swelling, crepitus, ankle and subtalar range of motion, talar tilt, and anterior drawer signs. ${ }^{4,5,13}$ It is important to compare the injured ankle to the uninjured ankle. While they are uncommon, the physician should rule out sensory abnormalities and muscle atrophy. The anterior talofibular ligament is the most commonly injured ligament with inversion sprains. ${ }^{4,6,13}$ A positive anterior drawer test is elicited when gentle traction of the heel causes pain and laxity (Figure 4A). It is the most appropriate clinical test for evaluation of lateral ankle ligament laxity. ${ }^{1}$ A positive talar tilt test occurs when the calcaneus is abducted and everted into the valgus position, resulting in laxity and pain, and it indicates that there has been concomitant injury to the calcaneofibular ligament along with the anterior talofibular ligament (Figure 4B). ${ }^{1}$

\section{Imaging}

Common imaging modalities for ankle injuries include plain film radiography, ultrasound, CT, and
Figure 4. The anterior drawer test (A) and talar tilt test (B) assess injury to the anterior talofibular ligament. A: With the patient in the sitting position and the knee flexed, the examiner stabilizes the distal leg with one hand while cupping the other hand around the ankle and applying force, moving the talus anteriorly. If ligament laxity is noted, the test is positive, consistent with rupture of the anterior talofibular ligament. The examiner must compare both ankles. B: With the patient in the sitting position and the ankle in plantar flexion, the examiner stabilizes the distal leg with one hand while applying force to the hindfoot with the other hand, slowly inverting the foot. If ligament laxity and pain is noted, the test is positive, consistent with an anterior talofibular ligament tear.

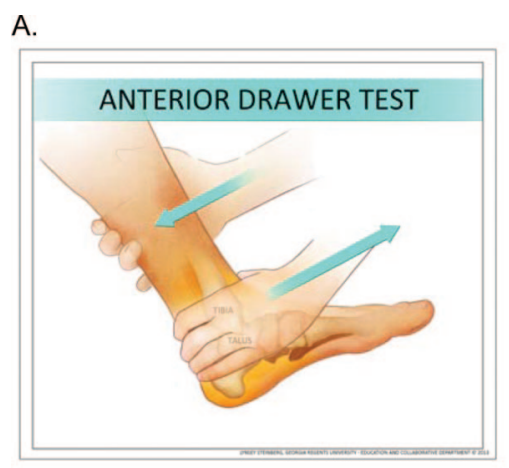

B.

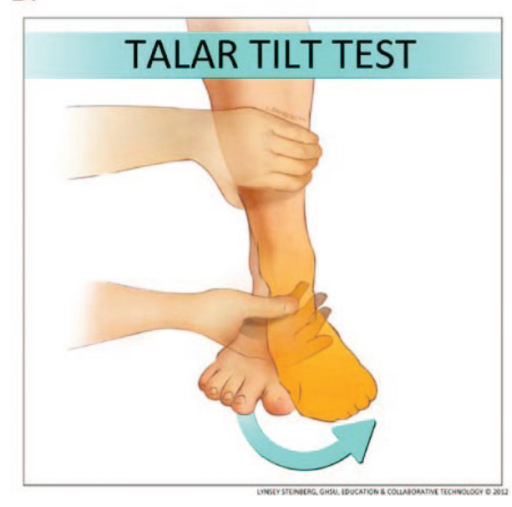

MRI; however, in the acute setting, to rule out a fracture, the Ottawa ankle rules should be used to assist in the clinical decision to obtain plain radiographs $^{1,4}$ (Figure 5). A systematic review of 27 studies involving 15,581 patients reported that the pooled negative likelihood ratio for ankle fractures was 0.08 (95\% confidence interval, $0.03-0.18){ }^{14}$ The Ottawa ankle rules have excellent sensitivity (almost 100\%) and are an accurate instrument for excluding fractures requiring immediate operative management of the ankle while reducing the num- 
Figure 5. Ottawa ankle rules are useful to assess whether radiographs are warranted: pain in the mid-foot zone and any one of the following: bony tenderness at base of the fifth metatarsal, or bony tenderness at navicular bone, or inability to bear weight in emergency department for 4 steps; or pain in the malleolar zone and any one of the following: bony tenderness along the distal $6 \mathrm{~cm}$ of the posterior edge of the tibia or tip of medial malleolus or bony tenderness along the distal $6 \mathrm{~cm}$ of posterior edge of the fibula or the tip of lateral malleolus or an inability to bear weight in the emergency department for 4 steps.

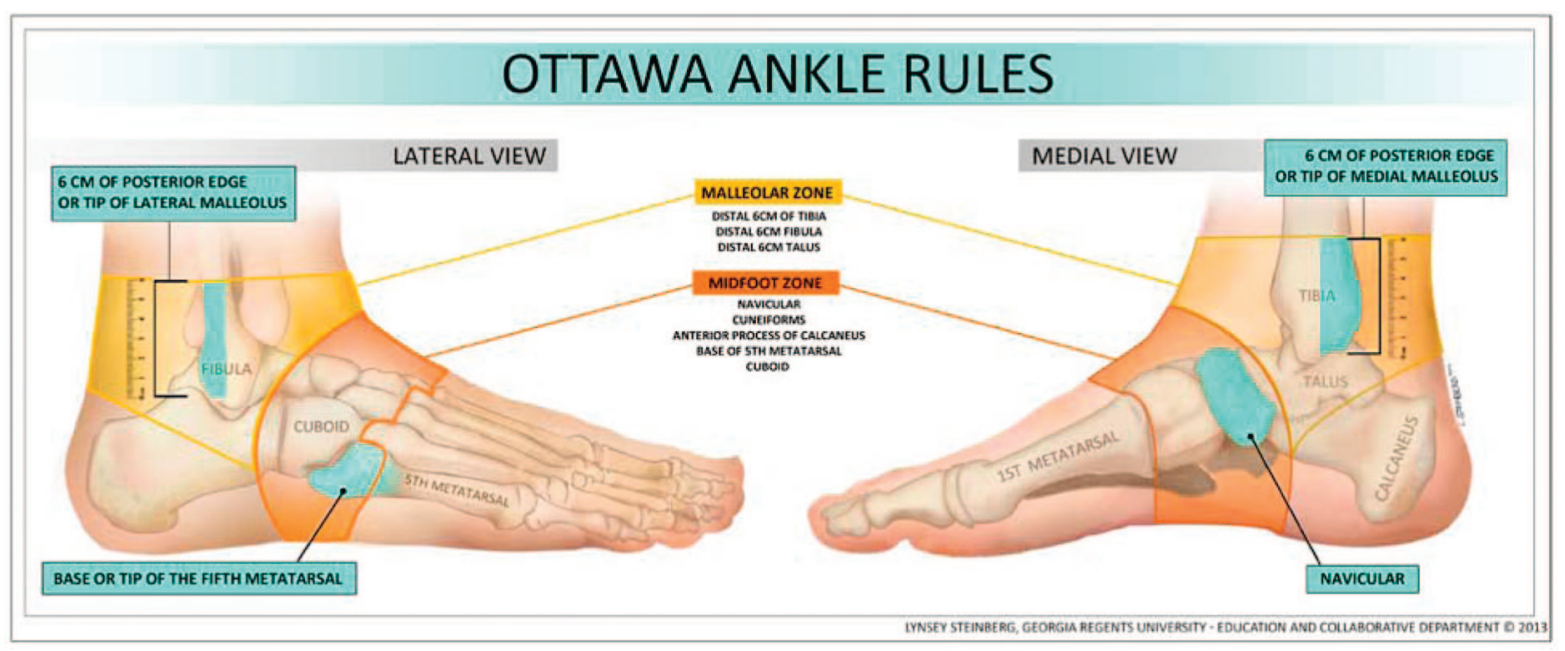

ber of unnecessary radiographs by $30 \%$ to $40 \%$ (Figure 5). ${ }^{14}$ A fifth metatarsal fracture is associated with a PBT injury where the PBT inserts on the proximal fifth metatarsal. The first-line imaging modality is ultrasound since it is less expensive than MRI and has comparable sensitivity (100\%) and specificity (85\%), positive likelihood ratio (6.7), and negative likelihood ratio (0). ${ }^{2,15}$ Ultrasound is dynamic, allowing for visualization of subluxation, and it may also be used for guided cortisone injections. $^{2}$ When an occult facture is suspected, CT is the preferred diagnostic test after negative plain film radiographs and persistent ankle pain. MRI is the preferred "gold standard" for ankle pain and instability when CT scan and radiograph are negative (sensitivity, 92\%; specificity, $83 \%$; positive likelihood ratio, 5.4; negative likelihood ratio, $0.1){ }^{1}$ MRI may visualize the contrast between tendon, subcutaneous tissue, and bone; however, MRI is costly, not easily available, and should be limited to patients with chronic ankle instability after previous diagnostic evaluations have failed to determine the etiology of symptoms. ${ }^{1,10}$

\section{Treatment}

Initial supportive therapy is important and may enhance the healing process and avoid chronic ligamentous laxity and surgery. This includes non- steroidal antiinflammatory drugs (NSAIDs), rest, ice, compression, elevation, and early mobilization. ${ }^{6}$ Topical NSAIDs may provide effective symptom relief with fewer side effects compared with oral NSAIDs. ${ }^{3}$ The combination of NSAIDs with cryotherapy (icing) is more effective than either therapy alone for acute ankle injuries. ${ }^{1}$ Inadequate supportive therapy of ankle sprains in the acute setting is associated with poor outcomes and chronic symptoms. ${ }^{6}$ Other treatment options include a Medrol dose pack or corticosteroid injection; however, there is no high-quality evidence to support the use of these measures in peroneal injuries.

Physical therapy is a good option for many patients because exercise maintains range of motion, assists with lymphatic drainage, and improves proprioception. Stretching is important to recover full function and enhance the healing process. ${ }^{3,4} \mathrm{Im}$ mobilization with braces and casting is a noninvasive option to stabilize the ankle and provide added protection from further injury. Most sprains usually resolve within 2 to 6 weeks with supportive therapy; however, if symptoms do not resolve, additional diagnostic evaluation and surgical options should be considered.

Surgical options include debridement, tubularization, and, in severe cases, resection of the damaged 
tendon and tenodesis. However, surgical therapy is recommended only after all other nonsurgical methods have failed to control symptoms. Twelve weeks after surgery, patients can be released to limited physical activity with bracing. ${ }^{13}$ After 6 months, patients can potentially be released without any restrictions if their instability has improved significantly. However, some patients may still experience instability following surgery. ${ }^{9,13}$ Common complications of surgery include numbness, hyperesthesia, crepitus, pain, and instability. ${ }^{5,9,13}$ A Cochrane review of 20 randomized controlled trials involving $2262 \mathrm{pa}-$ tients found insufficient evidence to determine the relative effectiveness of surgery versus conservative therapy for lateral ankle injuries. ${ }^{16} \mathrm{We}$ found no evidence regarding the relative effectiveness of surgery versus conservative therapy for peroneal injuries.

\section{Conclusion}

In our patient, a running injury 20 years earlier caused her ankle pain and instability. She subsequently reinjured her ankle and complained of lateral ankle pain, swelling, and instability, resulting in a grade II split. Despite supportive therapy with NSAIDs, physical therapy, and bracing, her symptoms persisted. Primary care physicians should consider peroneal tendon injuries in patients with chronic lateral ankle pain and instability.

We thank Jammie Edwards, DO, Lindsay Blake, MLIS, AHIP, and Lynsey Steinberg MSMI.

\section{References}

1. Polzer H, Kanz KG, Prall WC, et al. Diagnosis and treatment of acute ankle injuries: development of an evidence-based algorithm. Orthop Rev (Pavia) 2012; 4:e5.

2. Philbin TM, Landis GS, Smith B. Peroneal tendon injuries. J Am Acad Orthop Surg 2009;17:306-17.

3. Tiemstra JD. Update on acute ankle sprains. Am Fam Physician 2012;85:1170-6.

4. Wolfe MW, Uhl TL, Mattacola CG, McCluskey LC. Management of ankle sprains. Am Fam Physician 2001;63:93-104.
5. Solakoglu C, Kiral A, Pehlivan O, Akmaz I, Arpacioglu MO, Kaplan H. Late-term reconstruction of lateral ankle ligaments using a split peroneus brevis tendon graft (Colville's technique) in patients with chronic lateral instability of the ankle. Int Orthop 2003;27:223-7.

6. Yang J Jr, Morscher MA, Weiner DS. Modified Chrisman-Snook repair for the treatment of chronic ankle ligamentous instability in children and adolescents. J Child Orthop 2010;4:561-70.

7. DiGiovanni BF, Fraga CJ, Cohen BE, Shereff MJ. Associated injuries found in chronic lateral ankle instability. Foot Ankle Int 2000;21:809-15.

8. Strauss JE, Forsberg JA, Lippert FG 3rd. Chronic lateral ankle instability and associated conditions: a rationale for treatment. Foot Ankle Int 2007;28: 1041-4.

9. Sammarco GJ, DiRaimondo CV. Surgical treatment of lateral ankle instability syndrome. Am J Sports Med 1988;16:501-11.

10. Bonnin M, Tavernier T, Bouysset M. Split lesions of the peroneus brevis tendon in chronic ankle laxity. Am J Sports Med 1997;25:699-703.

11. Miura K, Ishibashi Y, Tsuda E, Kusumi T, Toh S. Split lesions of the peroneus brevis tendon in the Japanese population: an anatomic and histologic study of 112 cadaveric ankles. J Orthop Sci 2004;9: 291-5.

12. Athavale SA, Swathi, Vangara SV. Anatomy of the superior peroneal tunnel. J Bone Joint Surg Am 2011;93:564-71.

13. Colville MR, Grondel RJ. Anatomic reconstruction of the lateral ankle ligaments using a split peroneus brevis tendon graft. Am J Sports Med 1995;23: 210-3.

14. Bachmann LM, Kolb E, Koller MT, Steurer J, ter Riet G. Accuracy of Ottawa ankle rules to exclude fractures of the ankle and mid-foot: systematic review. BMJ 2003;326:417.

15. Grant TH, Kelikian AS, Jereb SE, McCarthy RJ. Ultrasound diagnosis of peroneal tendon tears. A surgical correlation. J Bone Joint Surg Am 2005;87: 1788-94.

16. Kerkhoffs GM, Handoll HH, de Bie R, Rowe BH, Struijs PA. Surgical versus conservative treatment for acute injuries of the lateral ligament complex of the ankle in adults. Cochrane Database Syst Rev 2007;(2):CD000380. 